\title{
Deference and Hierarchy in International Regime Complexes-ERRATUM
}

\author{
Tyler Pratt
}

doi: 10.1017/S0020818318000164. Published by Cambridge University Press, 6 May 2018.

In the original publication of this article, Table 3 was missing asterisks from several coefficients indicating statistical significance. The corrected Table 3 is replicated below:

TABLE 3. Effect of state power and functional efficiency variables on institutional deference

\begin{tabular}{|c|c|c|c|}
\hline & \multicolumn{3}{|c|}{ Dependent variable: Institutional Deference } \\
\hline & (1) & (2) & (3) \\
\hline MAJOR POWER DIFFERENCE & $\begin{array}{l}0.218^{* * *} \\
(0.055)\end{array}$ & $\begin{array}{l}0.218^{* * *} \\
(0.042)\end{array}$ & $\begin{array}{l}0.200^{* * * *} \\
(0.040)\end{array}$ \\
\hline WEIGHTED VOTING & $\begin{array}{l}0.698 * * \\
(0.293)\end{array}$ & $\begin{array}{c}0.428^{*} \\
(0.258)\end{array}$ & $\begin{array}{l}0.987 * * * \\
(0.174)\end{array}$ \\
\hline TECHNICAL IO & $\begin{array}{c}-0.088 \\
(0.340)\end{array}$ & $\begin{array}{c}-0.027 \\
(0.251)\end{array}$ & $\begin{array}{l}0.622 * * \\
(0.283)\end{array}$ \\
\hline BINDING-TECHNICAL PAIR & $\begin{array}{l}0.926 * * * \\
(0.316)\end{array}$ & $\begin{array}{c}0.325 \\
(0.275)\end{array}$ & $\begin{array}{l}0.774 * * * \\
(0.152)\end{array}$ \\
\hline DECISION-MAKING DIFFERENCE & $\begin{array}{l}0.298 * * \\
(0.145)\end{array}$ & $\begin{array}{c}0.208 \\
(0.141)\end{array}$ & $\begin{array}{l}0.328 * * \\
(0.144)\end{array}$ \\
\hline UN IDEAL POINT DIFFERENCE & & $\begin{array}{c}-0.161 \\
(0.277)\end{array}$ & $\begin{array}{c}-1.090 * * * \\
(0.250)\end{array}$ \\
\hline IO BUDGET & & $\begin{array}{c}-0.040 \\
(0.039)\end{array}$ & $\begin{array}{c}-0.071 \\
(0.043)\end{array}$ \\
\hline REGIONAL-GLOBAL IO PAIR & & $\begin{array}{r}-0.475 \\
(0.327)\end{array}$ & $\begin{array}{l}0.804 * * \\
(0.320)\end{array}$ \\
\hline RECIPROCITY & & $\begin{array}{l}2.133^{* * *} \\
(0.206)\end{array}$ & $\begin{array}{l}0.851 * * \\
(0.233)\end{array}$ \\
\hline ISSUE AREA FE & $\checkmark$ & $\checkmark$ & $\checkmark$ \\
\hline $\begin{array}{l}\text { IO DYAD FE } \\
\text { Observations }\end{array}$ & 3,718 & 3,343 & $\begin{array}{c}\sqrt{ } \\
3,343\end{array}$ \\
\hline
\end{tabular}

Notes: Results of generalized linear models estimating the effect of member state power and IO characteristics on directed institutional deference. All models include controls for membership overlap, an indicator for nested IOs, and a cubic polynomial for time (not shown). Statistical significance is denoted by: $* p<0.1 ; * * p<0.05 ; * * *<0.01$. 
762 International Organization

The publisher regrets this error.

\section{Reference}

Pratt, Tyler. 2018. Deference and Hierarchy in International Regime Complexes. International Organization 72 (3):561-90. doi: 10.1017/S0020818318000164. 